\title{
Metabolomics study of esophageal adenocarcinoma
}

\author{
Jian Zhang, MS, ${ }^{\text {a }}$ Lingyan Liu, MS, ${ }^{\mathrm{b}}$ Siwei Wei, MS, ${ }^{\text {a }}$ G. A. Nagana Gowda, PhD, ${ }^{\text {a }}$ Zane Hammoud, MD, ${ }^{\mathrm{c}}$ \\ Kenneth A. Kesler, MD, ${ }^{\mathrm{d}}$ and Daniel Raftery, $\mathrm{PhD}^{\mathrm{a}}$
}

Objective: The objective of this study was to detect and evaluate reliable metabolite markers for screening and monitoring treatment of patients with esophageal adenocarcinoma (EAC) by studying metabolomics. The sensitivity and specificity of the study were evaluated not only for EAC but also for Barrett esophagus and highgrade dysplasia, which are widely regarded as precursors of EAC.

Methods: Profiles of metabolites in blood serum were constructed using nuclear magnetic resonance spectroscopy and statistical analysis methods. The metabolite biomarkers discovered were selected to build a predictive model that was then used to test the classifications accuracies.

Results: Eight metabolites showed significant differences in their levels in patients with cancer and in the control group on the basis of Student $t$ test. A partial least-squares discriminant analysis model built on these metabolites provided excellent classifications of patients with cancer and the control group, with the area under the receiver operating in a characteristic curve of $>0.85$ for both training and validation sample sets. Evaluated by the same model, the Barrett esophagus samples were of mixed classification, and the high-grade dysplasia samples were classified primarily as cancer samples. A pathway study indicated that altered energy metabolism and changes in the trochloroacetic acid cycle were the dominant factors in the biochemistry of EAC.

Conclusions: ${ }^{1} \mathrm{H}$ nuclear magnetic resonance-based metabolite profiling analysis was shown to be an effective approach to differentiating between patients with EAC and healthy subjects. Good sensitivity and selectivity were shown by using the 8 metabolite markers discovered to predict the classification of samples from the healthy control group and the patients with the disease. Serum metabolic profiling may have potential for early diagnosis of EAC and may enhance our understanding of its mechanisms. (J Thorac Cardiovasc Surg 2011;141:469-75)

\section{Supplemental material is available online.}

Esophageal cancer (EC) is a leading cause of death from cancer worldwide. The two principal types of EC, squamous cell carcinoma and adenocarcinoma, are relatively uncommon in the United States, composing approximately $1 \%$ of all cancers. However, the incidence of adenocarcinoma is rising at a rapid rate. According to a report from the American Cancer Society, 12,300 new cases and 12,100 deaths were reported in $2000,{ }^{1}$ and the corresponding numbers for 2009 were 16,470 and 14,530 , respec-

\footnotetext{
From the Department of Chemistry, ${ }^{\mathrm{a}}$ Weldon School of Biomedical Engineering, ${ }^{\mathrm{b}}$ Purdue University, West Lafayette, Ind; Department of Cardiothoracic Surgery, ${ }^{c}$ Henry Ford Hospital, Detroit, Mich; and Department of Surgery, ${ }^{\mathrm{d}}$ Thoracic Surgery Division, Indiana University School of Medicine, Indianapolis, Ind.

Financial support: Purdue University's Center for Cancer Research and the Oncological Sciences Center in Discovery Park at Purdue.

Disclosures: Authors have nothing to disclose with regard to commercial support.

Received for publication April 19, 2010; revisions received July 12, 2010; accepted

for publication Aug 1, 2010; available ahead of print Sept 29, 2010.

Address for reprints: Zane Hammoud, MS, Department of Cardiothoracic Surgery,

Henry Ford Hospital, K-14, 2799 West Grand Boulevard, Detroit, MI 48202

(E-mail: zhammou1@hfhs.org).

$0022-5223 / \$ 36.00$

Copyright (c) 2011 by The American Association for Thoracic Surgery

doi:10.1016/j.jtcvs.2010.08.025
}

tively. ${ }^{2}$ The 5 -year survival rates of those with localized and all stages combined are $34 \%$ and $17 \%$, respectively. ${ }^{2}$ Moreover, currently there is no reliable method of early detection or of prediction of treatment outcome.

Barrett's esophagus (BE), high-grade dysplasia (HGD), and invasive cancer are thought to compose a multistep process in the development of esophageal adenocarcinoma (EAC) ${ }^{3}$ HGD has been considered to be the immediate precursor of invasive adenocarcinoma. ${ }^{4}$ Most patients with HGD are usually bearing or developing cancer, ${ }^{5}$ so HGD has been regarded as a marker of progression to carcinoma. ${ }^{6}$ However, no intervention currently in existence prevents the progression of BE or HGD to esophageal cancer. ${ }^{5}$ The traditional methods of diagnosing EC include endoscopy and barium swallow, ${ }^{7}$ but the poor specificity and sensitivity of these methods results in the detection of EC only at advanced stages. Recently, prognostic and predictive protein and genetic markers have been introduced to aid in the diagnosis of EC. ${ }^{89}$ However, markers effective at a potentially curative stage are lacking.

Metabolomics, a growing field in systems biology, offers a powerful and promising approach to identifying valuable biomarkers. Metabolomics (or metabolite profiling) describes the study of concentrations and fluxes of low molecular weight metabolites present in biofluids or tissues that provide detailed information about biologic systems and their current status. ${ }^{10,11}$ The information-rich analytic 


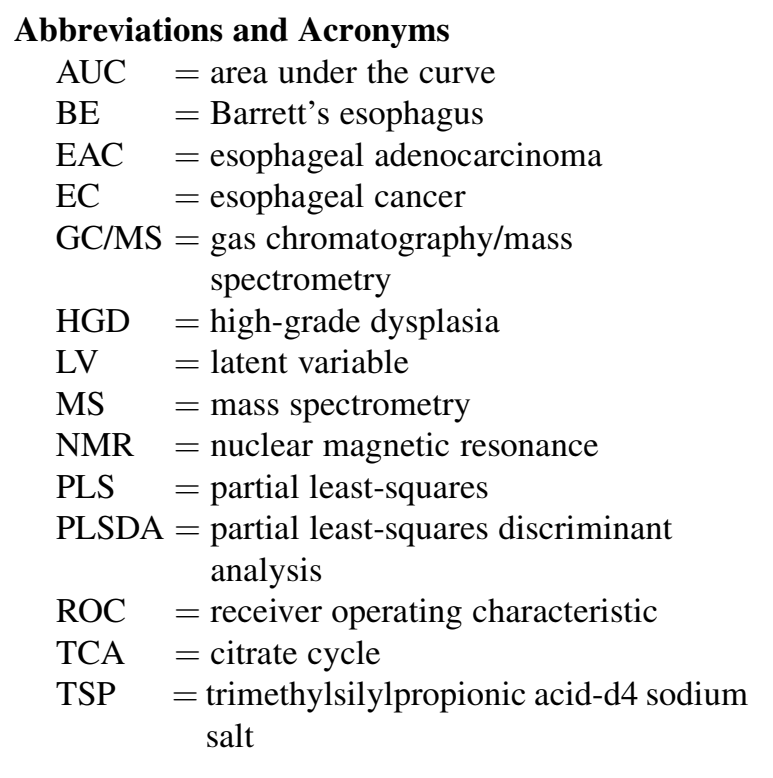

techniques of nuclear magnetic resonance (NMR) spectroscopy and mass spectrometry (MS) combined with multivariate statistical analyses are the premier methods for metabolomics-based studies. ${ }^{12}$ Various diseases, including but not limited to cancers, diabetes, inborn errors of metabolism, and coronary heart disease, have been explored using metabolomics as a tool, and a number of putative biomarkers have been detected and evaluated with the goal of improved diagnoses, assessment of risk, and even prediction of therapy outcomes. ${ }^{11}$ A recent example is the identification of sarcosine as a potential marker for prostate tumor aggressiveness; it was discovered using a metabolomics approach. ${ }^{13}$ However, few metabolomics studies of EC have been reported to date. Recently, Shen and coworkers reported 20 metabolite markers that were detected in fresh tumor tissue and corresponding normal esophageal mucosa by using gas chromatography/mass spectrometry ( $\mathrm{GC} /$ MS). ${ }^{14}$ To decrease the mortality rate of patients with esophageal cancer, development of early diagnostic methods, especially the exploitation of biomarkers that offer high sensitivity and specificity, is still in great demand. In addition, information provided by metabolomics in the area of biology may be useful in further understanding the biology of the disease.

In this study, ${ }^{1} \mathrm{H}$ NMR and multivariate statistical analysis were employed to detect molecular changes in human blood serum samples by comparing the metabolic profiles of patients with BE, HGD, and EAC as well as of normal controls in an attempt to identify a metabolite profile of EAC. We also attempted to identify a set of putative markers that may be useful in understanding the pathogenesis of EAC.

\section{MATERIALS AND METHODS \\ Chemicals}

Deuterium oxide $\left(\mathrm{D}_{2} \mathrm{O}, 99.9 \% \mathrm{D}\right)$ was purchased from Cambridge Isotope Laboratories, Inc. (Andover, Mass), and trimethylsilylpropionic acidd4 sodium salt (TSP) was purchased from Sigma-Aldrich (Milwaukee, Wis). All chemical reagents were analytic grade.

\section{Serum Sample Collection and Storage}

All work was conducted under a protocol approved by the Indiana University School of Medicine and Purdue University Institutional Review Board. All subjects included in the study provided informed consent according to institutional guidelines. All samples were collected when subjects were in the fasting state. Whole blood samples were collected from patients with histologically documented $\mathrm{BE}(\mathrm{n}=5)$, BE with HGD $(\mathrm{n}=11)$, and adenocarcinoma $(\mathrm{n}=68)$. Blood samples from 34 healthy volunteers served as controls. Each blood sample was allowed to clot for 45 minutes and then was centrifuged at $2000 \mathrm{rpm}$ for 10 minutes. The serum was collected, and an aliquote was put into in a separate vial and stored at $-80^{\circ} \mathrm{C}$ until use.

\section{${ }^{1}$ H NMR and Statistical Analysis}

Samples were prepared by mixing $200 \mu \mathrm{L}$ serum with $330 \mu \mathrm{L} \mathrm{D}_{2} \mathrm{O}$. A $60-\mu \mathrm{L}$ solution of TSP $(0.12 \mathrm{mg} / \mathrm{mL})$ sealed in a separate capillary was used as an internal standard, which acted as the frequency standard $(\delta=0.00)$. A Bruker DRX 500-MHz spectrometer equipped with a room-temperature hydrogen cyanide probe was used to acquire 1-dimensional ${ }^{1} \mathrm{H}$ NMR spectra. The water signal was suppressed using a standard 1-dimensional Carr-Purcell-Meiboom-Gill pulse sequence coupled with water presaturation. For each spectrum, 64 transients were collected, and $16 \mathrm{~K}$ data points were acquired using a spectral width of $6000 \mathrm{~Hz}$. An exponential weighting function corresponding to $1 \mathrm{~Hz}$ line broadening was applied to the free-induced decay before Fourier transformation. Phasing and baseline correction were applied using Bruker TopSpin software.

To remove the errors resulting from the small fluctuations of chemical shifts due to $\mathrm{pH}$ or ion concentration variations, NMR spectral regions were binned to $4 \mathrm{~K}$ buckets of equal width $(1.5 \mathrm{~Hz})$. Each spectrum was aligned to the methyl peak of alanine at $1.48 \mathrm{ppm}$, and was normalized using the integrated TSP signal. Spectral regions within the range of 0.3 to 10 $\mathrm{ppm}$ were used after deleting the region containing the water resonance and urea signal ( 4.5 to $6 . \mathrm{ppm})$.

To visualize the differences between spectra better, partial least-squares (PLS), a robust supervised method to detect subtle changes between group variations, was employed. PLS fits data matrices $X$ (which consists of NMR spectra) and $Y$ (which is set to 1 for cancer and 0 for control) and recasts these data as score plots and loading plots. The NMR spectral signals, or variables, were autoscaled (by subtracting the mean value of each variable and dividing by its standard deviations) prior to all statistical analyses. The score plot shows the possible relationships (or clustering) among the samples to estimate the classification; each orthogonal axis is named a latent variable (LV). The corresponding loading plot of each LV contains the weight or contribution of each variable in the modeling. To explore potential biomarker candidates, univariate analysis was performed by calculating the $P$ value (unpaired Student $t$ test) and a Benjamini-Hochberg correction was followed in order to control false discovery errors originating from multiplicity. ${ }^{15}$

Subsequently, a partial least-squares discriminant analysis (PLS-DA) model was built to evaluate the biomarker candidates when combined as a metabolite profile. Predictions were made visually using a Y-predicted scatter plot with a cut-off value chosen for potential class membership. The NMR data were imported into Matlab (R2008a; MathWorks, Natick, Mass) and installed using a PLS toolbox (version 4.1; Eigenvector Research, Inc.) for PLS and PLS-DA analysis. 


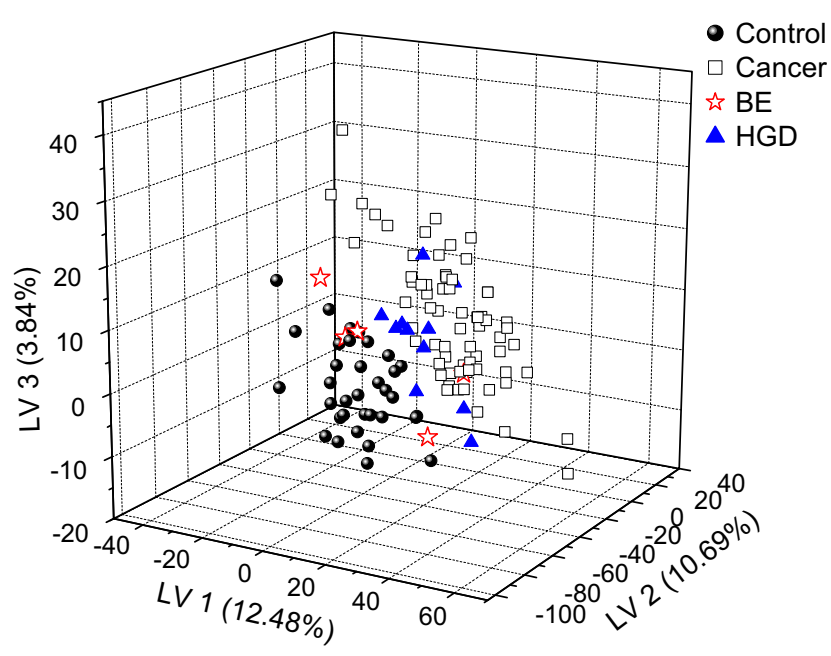

FIGURE 1. PLS score plot based on the ${ }^{1} \mathrm{H}$ NMR spectra of normal subjects, BE, HGD and esophageal cancer patients.

\section{RESULTS}

Representative ${ }^{1} \mathrm{H}$ NMR spectra of samples from the control group and the esophageal cancer group are shown in Figure E1, along with a difference spectrum that shows only a moderate amount of difference among the spectra. However, cancerous and control samples could be clearly separated when contributions from many signals in the spectra were combined, as illustrated in the PLS score plot shown in Figure 1. Also, and this is promising, the BE and HGD samples appeared in the middle of the cluster of control and cancer samples in the same plot. HGD samples were generally closer to the cluster of the cancer samples. However, examination of the PLS loadings indicated that a large number of low-level signals contributed and few easily detected signals dominated.

To explore and evaluate potential biomarker candidates better, all the control and esophageal cancer samples were randomly and equally divided into 2 groups: G1, the training group; and G2, the test group (Table 1). For the G1 samples, $P$ values were calculated for all data points. There were 19 spectral regions that showed a statistical difference between cancer and control (uncorrected $P$ value $<.05$ ); they were identified and integrated. $P$ values along with a Benjamini-Hochberg correction were calculated for the integrated peaks, and 14 peaks with a corrected $P$ value $<.05$ were short-listed (Table E1). According to the literature, ${ }^{16}$ these peaks belonged to 8 potential biomarker candidates, which were identified as $\beta$-hydroxybutyrate, lysine, glutamine, citrate, creatinine, lactate, glucose, and an unknown molecule. The same metabolite peaks in G2 were also integrated. Fold changes were calculated by dividing the average cancer values by the average control values. All the metabolite concentrations collected were higher in the cancer samples, as shown in Table 2.
TABLE 1. Clinicopathologic characteristics of patients with esophageal cancer

\begin{tabular}{|c|c|c|c|c|c|c|c|}
\hline \multicolumn{4}{|c|}{ Training group (G1) } & \multicolumn{4}{|c|}{ Test group (G2) } \\
\hline $\begin{array}{c}\text { Patient } \\
\text { no. }\end{array}$ & Age & Sex & cStage & $\begin{array}{c}\text { Patient } \\
\text { no. }\end{array}$ & Age & Sex & cStage \\
\hline 1 & 69 & $\mathrm{~F}$ & T3N1 & 1 & 66 & M & T3N1 \\
\hline 2 & 69 & M & T1N0 & 2 & 60 & M & T3N1 \\
\hline 3 & 63 & M & T3N1 & 3 & 64 & M & $\mathrm{T} 3 \mathrm{~N} 1$ \\
\hline 4 & 71 & M & T1N0 & 4 & 58 & $\mathrm{~F}$ & T4N1 \\
\hline 5 & 77 & M & T3N1 & 5 & 68 & M & M \\
\hline 6 & 49 & M & M & 6 & 46 & M & M1a \\
\hline 7 & 78 & M & T3N1 & 7 & 78 & M & T3N1 \\
\hline 8 & 61 & M & M & 8 & 78 & M & T1N0 \\
\hline 9 & 69 & M & T1N0 & 9 & 66 & M & T3N1 \\
\hline 10 & 57 & $\mathrm{~F}$ & T1N0 & 10 & 53 & M & T2NO \\
\hline 11 & 65 & M & T3N1M1a & 11 & 54 & $\mathrm{~F}$ & T3N1M1a \\
\hline 12 & 60 & $\mathrm{~F}$ & $\mathrm{~T} 2 \mathrm{~N} 0$ & 12 & 62 & M & T1N0 \\
\hline 13 & 68 & M & unk & 13 & 59 & $\mathrm{~F}$ & unk \\
\hline 14 & 51 & M & unk & 14 & 75 & M & T3N0 \\
\hline 15 & 47 & $\mathrm{~F}$ & T3NO & 15 & 78 & M & $\mathrm{T} 2 \mathrm{~N} 1$ \\
\hline 16 & 56 & M & T2N1M1a & 16 & 72 & M & T3N0 \\
\hline 17 & 71 & M & $\mathrm{T} 2 \mathrm{Nx}$ & 17 & 77 & $\mathrm{~F}$ & T3N0 \\
\hline 18 & 58 & M & unk & 18 & 72 & M & unk \\
\hline 19 & 91 & M & T3N0 & 19 & 82 & $\mathrm{~F}$ & T3N1M1b \\
\hline 20 & 74 & M & T3N1M1a & 20 & 58 & M & T3N1 \\
\hline 21 & 62 & M & T3N1 & 21 & 67 & M & T3N1 \\
\hline 22 & 64 & M & $\mathrm{T} 3 \mathrm{~N} 1$ & 22 & 67 & M & T3N1M1 \\
\hline 23 & 53 & M & T2N1M1 & 23 & 77 & M & T1N0 \\
\hline 24 & 54 & M & T2N1M1a & 24 & 62 & M & T3N1 \\
\hline 25 & 64 & $\mathrm{~F}$ & T3N1 & 25 & 67 & M & $\mathrm{T} 3 \mathrm{Nx}$ \\
\hline 26 & 72 & M & T3N1M1a & 26 & 54 & M & T3N1 \\
\hline 27 & 64 & M & T3N1 & 27 & 72 & M & T3N1 \\
\hline 28 & 71 & M & T2N0 & 28 & 67 & M & T3N1M1 \\
\hline 29 & 65 & M & T3N0 & 29 & 66 & M & unk \\
\hline 30 & 57 & M & T3N1M1a & 30 & 64 & M & T2N1M1 \\
\hline 31 & 78 & M & T4N1 & 31 & 71 & M & T3N1M1 \\
\hline 32 & 66 & M & $\mathrm{T} 2 \mathrm{~N} 1$ & 32 & 68 & M & $\mathrm{T} 3$ \\
\hline 33 & 74 & M & T3N3M1 & 33 & 62 & F & $\mathrm{T} 2 \mathrm{~N} 1$ \\
\hline 34 & 63 & M & T3N1 & 34 & 61 & M & $\mathrm{T} 2 \mathrm{Nx}$ \\
\hline
\end{tabular}

cStage, Clinical stage; $U N K$, unknown.

PLS-DA was then used to build a multivariate model to evaluate the potential biomarker candidates together. The 8 metabolites in the G1 samples were selected as the variables to build the PLS-DA model. Leave-one-out cross-validation was performed to obtain the best model and avoid overfitting. Three LVs were used and the cross-validation error was estimated to be $14.7 \%$. The model was then reapplied to the samples in G2. The PLS-DA score plots of G1 and $\mathrm{G} 2$ are shown in Figure 2, $A$, and 2, B, respectively. Most samples were well classified using a cut-off of 0.0855 . In Figure 2, $C$, and 2, $D$, receiver operating characteristic (ROC) curve analysis using the cross-validated predicted Y (predicted class) values was utilized to judge the sensitivity and specificity of the PLS-DA model. The area 
TABLE 2. Metabolites showing a significant separation of control samples from cancer samples in the training group and their fold change values

\begin{tabular}{lcc}
\hline \multicolumn{1}{c}{ Metabolite } & $\boldsymbol{P}$ value* & Fold change \\
\hline Glutamine & $3.02 \mathrm{E}-02$ & 1.10 \\
$\beta$-Hydroxybutyrate & $2.28 \mathrm{E}-05$ & 1.31 \\
Citrate & $3.31 \mathrm{E}-04$ & 1.26 \\
Unknown & $2.97 \mathrm{E}-05$ & 1.26 \\
Lysine & $9.63 \mathrm{E}-04$ & 1.10 \\
Creatinine & $2.19 \mathrm{E}-02$ & 1.23 \\
Lactate & $2.66 \mathrm{E}-03$ & 1.28 \\
$\alpha$-Glucose & $1.49 \mathrm{E}-04$ & 1.20
\end{tabular}

*Benjamini-Hochberg correction was used to control for possible false discovery resulting from the use of multiple variable comparison. In this case 19 variables were used.

under the curve (AUC) of G1 and G2 were 0.875 and 0.888 , respectively. The sensitivity and specificity for the detection of EC were $88 \%$ and $82 \%$ for G1 and $88 \%$ and $92 \%$ for $\mathrm{G} 2$, respectively.
Metabolic differences might act as a useful tool for grading tumors compared with the classical individual metabolite-based targeted analysis, so $P$ values and boxand-whisker plot studies were employed to try to use the metabolite profile to identify patients with early-stage disease. The $P$ value results of various group comparisons of control, BE, HGD, and cancer samples are listed in Table E2. The low $P$ values of the metabolites for all the control and cancer samples demonstrated that the levels of these molecules were statistically different. However, $P$ values between adjoining stages were always high, indicating that metabolite changes from one stage to the next stage were not significant.

Comparing HGD with controls, changes in the concentrations of citrate, creatinine, lactate, glucose, and the unknown species were notable according to the $P$ values; and the levels of $\beta$-hydroxybutyrate, lysine, and glutamine changed significantly between $\mathrm{BE}$ and cancer. The ranges

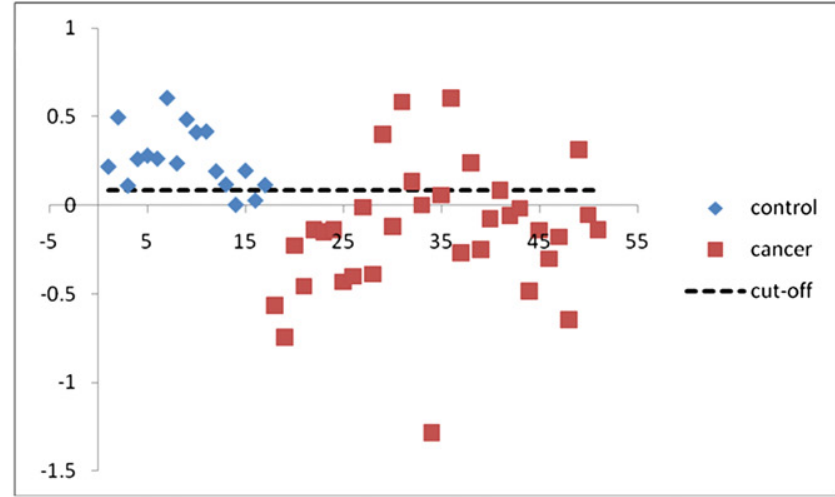

A

ROC Curve

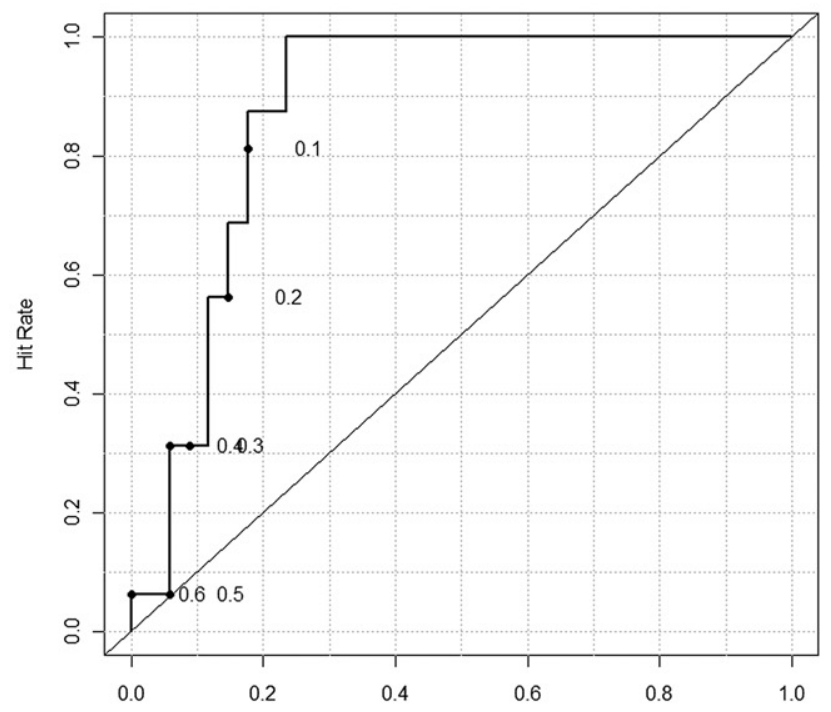

C

False Alarm Rate

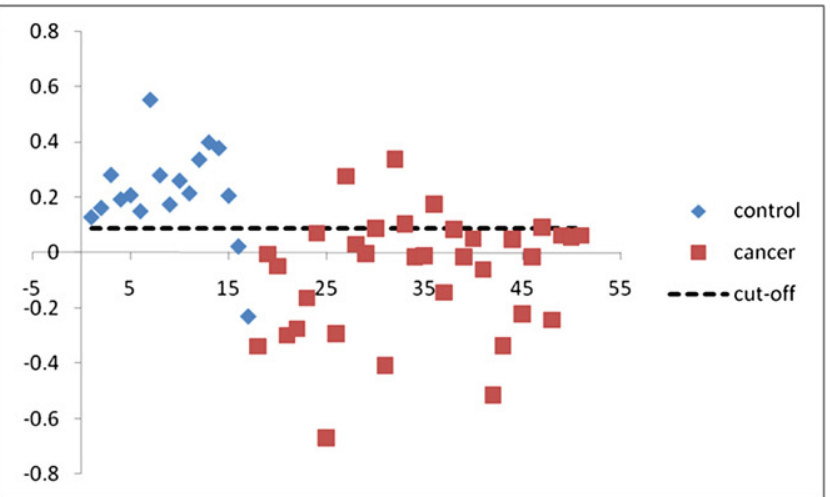

B

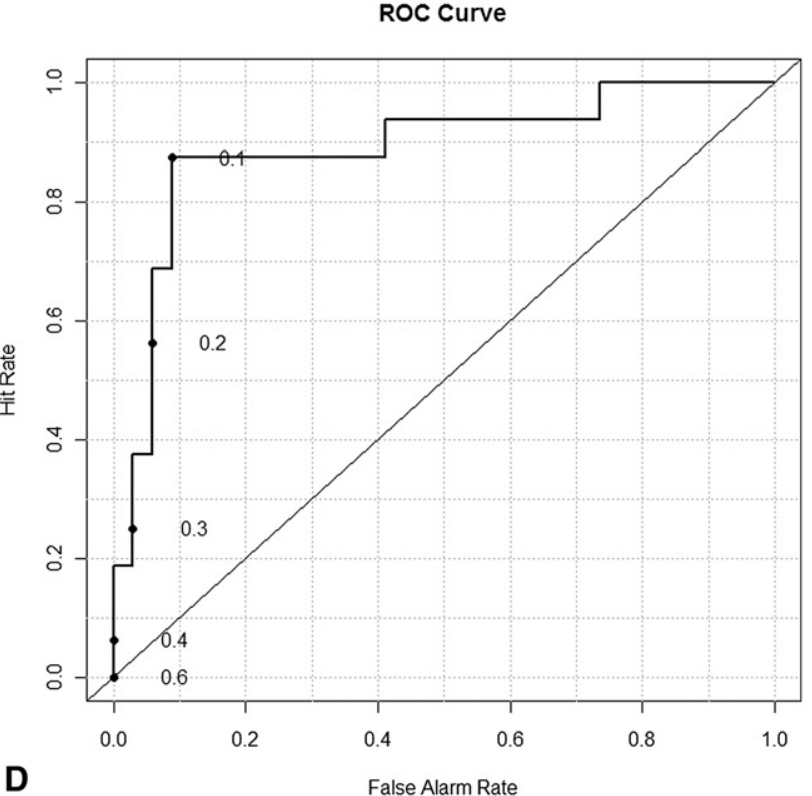

FIGURE 2. Results of the PLS-DA model from the 8 metabolite markers for (A) training group (G1) and (B) test group (G2), and ROC curves using the cross-validated predicted class values for (C) G1 and (D) G2. The AUC of G1 and G2 are 0.875 and 0.888 , respectively. 
of concentration of each metabolite in samples from control, HGD, and cancer are shown as box-and-whisker plots in Figure E2. The BE data were excluded from this plot because of the small number of samples. The same increasing trends of each metabolite showed in the plots from normal to HGD to cancer. For citrate, creatinine, lactate, and glucose, the changes between normal and HGD were greater than the changes between HGD and cancer, which were consistent with the $P$ value results. The possible effects of age and gender were also investigated in the cancer samples (Table E3). Large $P$ values were observed between male and female patients for each of the eight markers, indicating that gender is not likely to be a significant factor in the classification. Similarly, $P$ values comparing younger and older patients are also high, indicating that an age effect is also not likely to be present.

To further evaluate the BE and HGD samples, the same PLS-DA model used for predicting the control and cancer samples was applied, and the result is shown in Figure 3. $\mathrm{BE}$ samples gave a mixed result, and no confident conclusion could be made because of the small number of samples. However, 9 of 11 HGD samples were predicted to be cancer in this case, which supported the previous PLS result.

\section{DISCUSSION}

A metabolomics approach based on ${ }^{1} \mathrm{H}$ NMR coupled with multivariate statistical methods such as PLS or PLSDA and starting with a metabolite identified by employing a univariate statistical method ( $P$ values) provides a powerful approach to metabolic profiling of blood serum to differentiate patients with EAC from control subjects. Although the patients with esophageal cancers were easily distinguished from control subjects by PLS using autoscaling, when using other scaling methods (Pareto or log scaling), this approach was not very successful. This result is explained by the contribution of a number of low-intensity signals that can contribute to the classification, depending on the particular scaling method used. However, significant

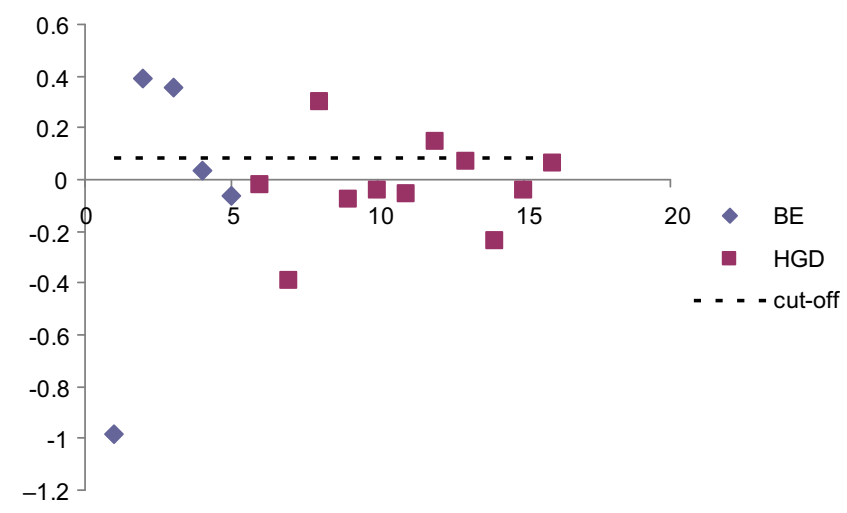

FIGURE 3. PLS-DA prediction from the model based on 8 metabolite markers for BE and HGD samples. noise also contributes to the low signals that are unidentifiable by standard NMR techniques. Using the same model without including the healthy controls failed to discriminate differing ages and genders of patients with EC (data not shown), which indicates that age and gender were probably not relevant parameters in the model. However, a targeted approach using 8 detected biomarker candidates (the vast majority of which can be identified easily) that exhibit low $P$ values appears to be a good starting place for multivariate model building. The model had high sensitivity and specificity for the prediction of EAC.

Altered pathways in EAC were identified based on the metabolites that showed significant concentration changes. Based on the Kyoto Encyclopedia of Genes and Genomes database (http://www.genome.jp/kegg/pathway.html), a simplified pathway map is shown in Figure 4. The upregulation of glucose and lactate, a common observation in the serum of cancer subjects, was detected in samples from patients with EC. The phenomenon of cancer cells' avidly taking up glucose and producing lactic acid under aerobic conditions was hypothesized as being aerobic glycolysis by Otto Warburg in $1924 .{ }^{17}$ The metabolization of glucose with a concomitant increase of lactate production has been regarded as a common trait in many rapidly proliferating cancers. A continuous supply of glucose is demanded by cancer cells to produce glycoproteins, triglycerides, and glycogen and as an important source of energy. High molar concentrations of lactate were indicated to be correlated with a high incidence of distant metastasis, even in an early stage of the disease. $\mathrm{Nu}$ merous recent reports support these data by demonstrating various biologic activities of lactate that can enhance the malignant behavior of cancer cells, including epithelial ovarian cancer, ${ }^{18}$ cervical cancer, ${ }^{19}$ colorectal cancer, ${ }^{20}$ and various primary carcinomas in the head, neck, and colorectal regions. ${ }^{21}$ Thus, glucose and lactate accumulation mirrors the higher energy demand of tumor malignancy. The average absolute concentration of glucose is above the normal range in cancer patients; however, its counterpart, lactate, is nevertheless roughly within the normal range, so these markers individually do not point to abnormality (Table E4).

The organic acids $\beta$-hydroxybutyrate and citrate were also found to be higher in patients with EAC compared to subjects without EAC. Increased amounts of $\beta$-hydroxybutyrate may be to the result of increased energy metabolism in the tumor, which results in large amounts of lactate being produced by the tumor. ${ }^{22}$ When lactate is abundant, the Cori cycle might not be able to convert lactate back into glucose in the liver, which results in the accumulation of acetyl-CoA and the citrate upregulation in the citrate cycle (TCA cycle) sequentially. ${ }^{23}$ If the acetyl-CoA is not well accommodated by the TCA cycle, ketogenesis takes place. As a ketone body, $\beta$-hydroxybutyrate is converted by $\beta$-hydroxybutyrate dehydrogenase, which results in increased levels of $\beta$-hydroxybutyrate. ${ }^{22}$ 


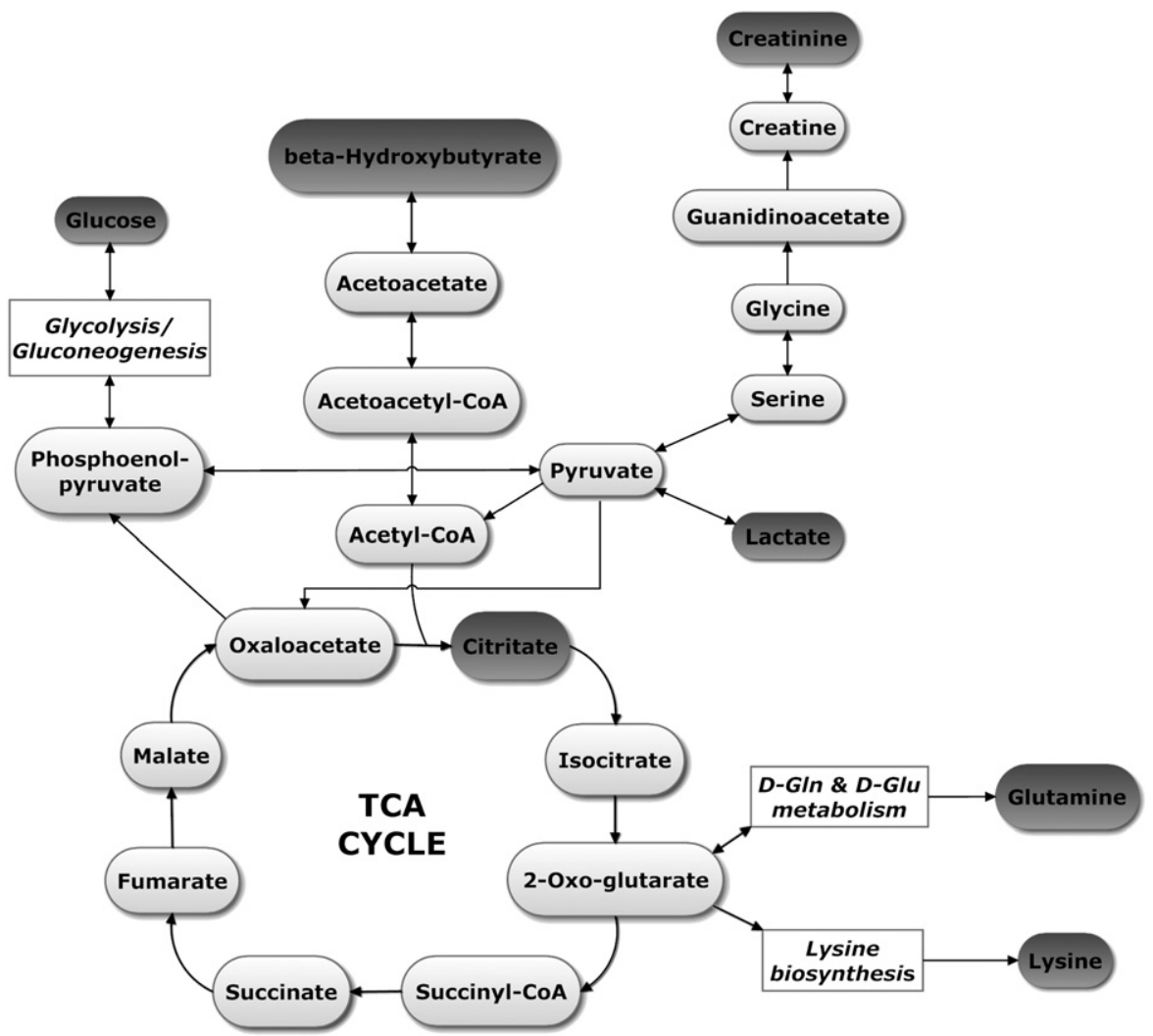

FIGURE 4. Simplified altered metabolism pathways for the most relevant metabolic differences between patients with esophageal cancer and control subjects. The metabolites in gray were the biomarkers observed as being upregulated in patients with esophageal cancer.

Higher levels of lysine, glutamine, and creatinine were also found in the serum of subjects with EC. This is consistent with the effects on the TCA cycle and lactate accumulation, which provide precursors for many compounds, including lysine, glutamine, and creatinine. It has been reported that the human hepatocellular carcinoma tumors produce levels of glutamine that are higher than those found in noninvolved adjacent liver tissues. ${ }^{24}$ Elevated levels of lysine are also in close agreement with previous findings in the extracellular fluid of patients with cerebral gliomas ${ }^{25}$ and colon carcinomas. ${ }^{26}$ Creatinine levels are known to be affected by various cancers, and they were recently observed to be high in a report analyzing tumor samples from patients with oral squamous cell carcinoma. ${ }^{27}$ Nevertheless, the absolute concentrations of creatinine and glutamine were also not particularly unusual (Table E4).

\section{Limitations}

A limitation of the current study is the fact that peaks generated by different species often overlap in ${ }^{1} \mathrm{H}$ NMR spectra, especially in the aliphatic region (1-5 ppm). As a result, potentially important compounds present at smaller concentrations may be overshadowed by larger peaks and may be hard to detect. As can be seen in the PLS analysis, a separation between patients with BE and HDG and the differentiation between them and controls without cancer are possible, but these separations rely on the detection of species that occur in lower concentrations. The combination of NMR with other analytic platforms such as MS will broaden the ability to detect lower-concentration biomarkers and, it is hoped, allow more reliable metabolite profiles to be constructed. ${ }^{12}$ Future work will focus on the use of LC/MS and GC/MS analyses to discover other potential biomolecules. The analysis of additional clinical samples may allow us to differentiate more successfully among BE, HGD, and EC as well as to provide information about staging, which is currently not possible.

\section{CONCLUSIONS}

${ }^{1} \mathrm{H}$ NMR-based metabolite profiling analysis has been shown to be an effective approach to differentiating patients with EAC from healthy subjects. HGD and BE were also evaluated using the profile developed from serum metabolites, and these conditions could be classified as falling between cancer and normal, as might be expected on the basis of their known relationship with the disease. We discovered 8 metabolite markers, including glucose, lactate, citrate, $\beta$-hydroxybutyrate, lysine, glutamine, creatinine, and one 
unknown metabolite, with good discriminating ability and low $P$ values. The results indicate that alteration of energy metabolism coupled with changes in TCA cycle were dominant in the biochemistry of EAC. Good sensitivity and selectivity were shown in the use of these markers to predict whether the samples came from the healthy control group or the subjects with disease. These results indicate the promise of this approach in developing a profile for earlier detection. The analysis of additional samples and the use of additional spectroscopic methods such as MS are expected to result in the foundation that will allow for an improved diagnostic assay.

\section{References}

1. Greenlee RT, Murray T, Bolden S, Wingo PA. Cancer statistics, 2000. CA Cancer J Clin. 2000;50:7-33.

2. Jemal A, Siegel R, Ward E, Hao Y, Xu J, Thun MJ. Cancer statistics, 2009. CA Cancer J Clin. 2009;59:225-49.

3. Reed MF, Tolis G Jr, Edil BH, Allan JS, Donahue DM, Gaissert HA, et al. Surgical treatment of esophageal high-grade dysplasia. Ann Thorac Surg. 2005;79: 1110-5.

4. Waxman I, Raju GS, Critchlow J, Antonioli DA, Spechler SJ. High-frequency probe ultrasonography has limited accuracy for detecting invasive adenocarcinoma in patients with Barrett's esophagus and high-grade dysplasia or intramucosal carcinoma: a case series. Am J Gastroenterol. 2006;101:1773-9.

5. Shaheen NJ, Richter JE. Barrett's oesophagus. Lancet. 2009;373:850-61.

6. Prasad GA, Wang KK, Halling KC, Buttar NS, Wongkeesong LM, Zinsmeister AR, et al. Utility of biomarkers in prediction of response to ablative therapy in Barrett's esophagus. Gastroenterology. 2008;135:370-9.

7. Javle M, Ailawadhi S, Yang GY, Nwogu CE, Schiff MD, Nava HR. Palliation of malignant dysphagia in esophageal cancer: a literature-based review. J Support Oncol. 2006;4:365-73, 79.

8. van Baal JW, Krishnadath KK. High throughput techniques for characterizing the expression profile of Barrett's esophagus. Dis Esophagus. 2008;21:634-40.

9. van Baal JW, Rygiel AM, Milano F, Anderson M, Bergman JJ, Spek CA, et al. Gene expression profile comparison of Barrett's esophagus epithelial cell cultures and biopsies. Dis Esophagus. 2008;21:628-33.

10. Nicholson JK, Lindon JC, Holmes E. "Metabonomics": understanding the metabolic responses of living systems to pathophysiological stimuli via multivariate statistical analysis of biological NMR spectroscopic data. Xenobiotica. 1999;29: 1181-9.
11. Gowda GA, Zhang S, Gu H, Asiago V, Shanaiah N, Raftery D. Metabolomics-based methods for early disease diagnostics. Expert Rev Mol Diagn. 2008;8:617-33.

12. Pan Z, Raftery D. Comparing and combining NMR spectroscopy and mass spectrometry in metabolomics. Anal Bioanal Chem. 2007;387:525-7.

13. Sreekumar A, Poisson LM, Rajendiran TM, Khan AP, Cao Q, Yu J, et al. Metabolomic profiles delineate potential role for sarcosine in prostate cancer progression. Nature. 2009;12:910-4.

14. Wu H, Xue R, Lu C, Deng C, Liu T, Zeng H, et al. Metabolomic study for diagnostic model of oesophageal cancer using gas chromatography/mass spectrometry. J Chromatogr B Analyt Technol Biomed Life Sci. 2009;877:3111-7.

15. Benjamini Y, Hochberg Y. Controlling the false discovery rate: a practical and powerful approach to multiple testing. J Roy Stat Soc.B. 1995;57:289-300.

16. Nicholson JK, Foxall PJ, Spraul M, Farrant RD, Lindon JC. $750 \mathrm{MHz} 1 \mathrm{H}$ and 1H-13C NMR spectroscopy of human blood plasma. Anal Chem. 1995;67: 793-811.

17. Warburg O, Posener K, Negelein E. On the metabolism of carcinoma cells. Biochemische Zeitschrift. 1924;152:309-44.

18. Odunsi K, Wollman RM, Ambrosone CB, Hutson A, McCann SE, Tammela J, et al. Detection of epithelial ovarian cancer using 1H-NMR-based metabonomics. Int J Cancer. 2005;113:782-8.

19. Walenta S, Wetterling M, Lehrke M, Schwickert G, Sundfor K, Rofstad EK, et al High lactate levels predict likelihood of metastases, tumor recurrence, and restricted patient survival in human cervical cancers. Cancer Res. 2000;60:916-21.

20. Qiu Y, Cai G, Su M, Chen T, Zheng X, Xu Y, et al. Serum metabolite profiling of human colorectal cancer using GC-TOFMS and UPLC-QTOFMS. J Proteome Res. 2009;8:4844-50.

21. Walenta S, Schroeder T, Mueller-Klieser W. Lactate in solid malignant tumors potential basis of a metabolic classification in clinical oncology. Curr Med Chem. 2004;11:2195-204.

22. Rantalainen M, Cloarec O, Beckonert O, Wilson ID, Jackson D, Tonge R, et al Statistically integrated metabonomic-proteomic studies on a human prostate cancer xenograft model in mice. J Proteome Res. 2006;5:2642-55.

23. Tisdale MJ. Cancer cachexia: metabolic alterations and clinical manifestations. Nutrition. 1997;13:1-7.

24. Yang Y, Li C, Nie X, Feng X, Chen W, Yue Y, et al. Metabonomic studies of human hepatocellular carcinoma using high-resolution magic-angle spinning $1 \mathrm{H}$ NMR spectroscopy in conjunction with multivariate data analysis. $J$ Proteome Res. 2007;6:2605-14.

25. Bianchi L, De Micheli E, Bricolo A, Ballini C, Fattori M, Venturi C, et al. Extracellular levels of amino acids and choline in human high grade gliomas: an intraoperative microdialysis study. Neurochem Res. 2004;29:325-34.

26. Denkert C, Budczies J, Weichert W, Wohlgemuth G, Scholz M, Kind T, et al. Metabolite profiling of human colon carcinoma-deregulation of TCA cycle and amino acid turnover. Mol Cancer. 2008;7:72.

27. Tiziani S, Lopes V, Gunther UL. Early stage diagnosis of oral cancer using $1 \mathrm{H}$ NMR-based metabolomics. Neoplasia. 2009;11:269-76. 


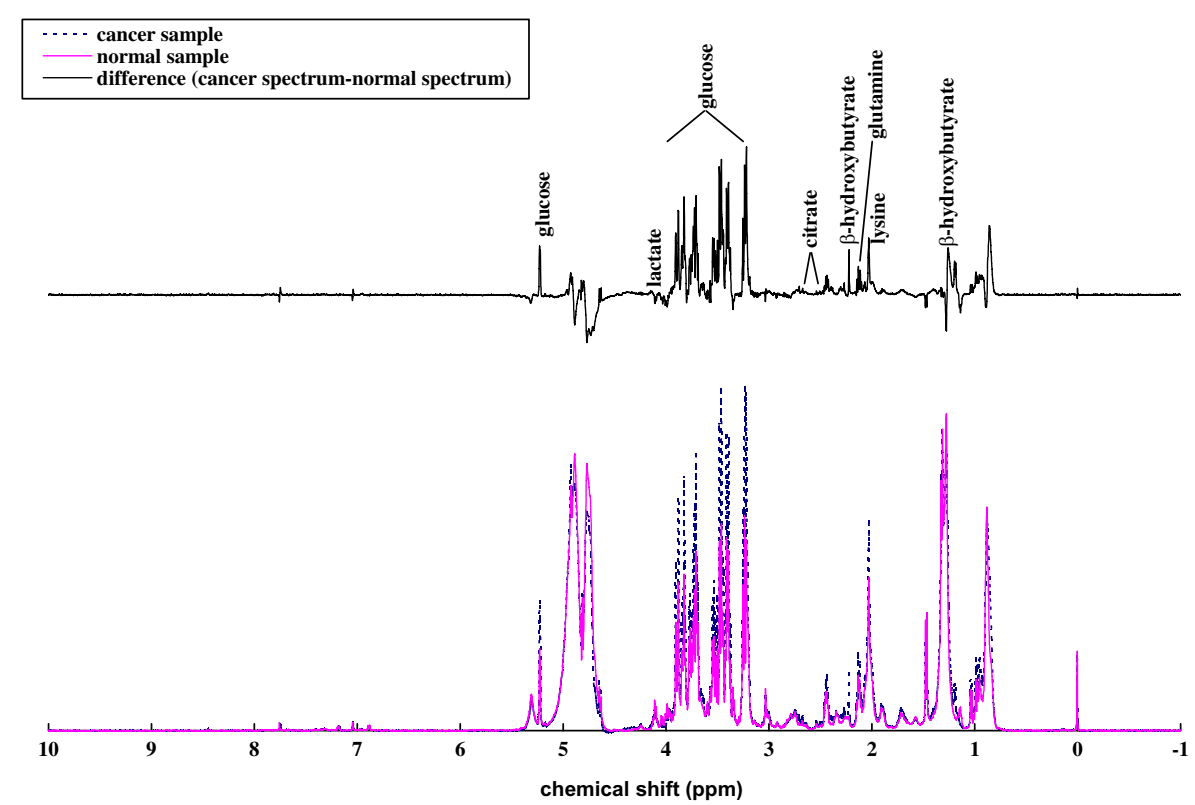

FIGURE E1. Typical ${ }^{1}$ H NMR CPMG serum spectra from a healthy volunteer and a patient with esophageal cancer (bottom), and their difference spectrum (top). 


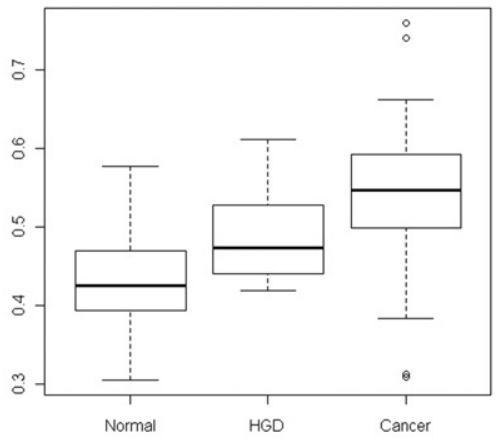

glutamine

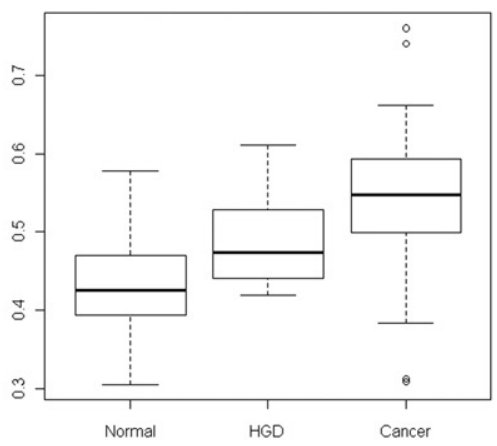

unknown

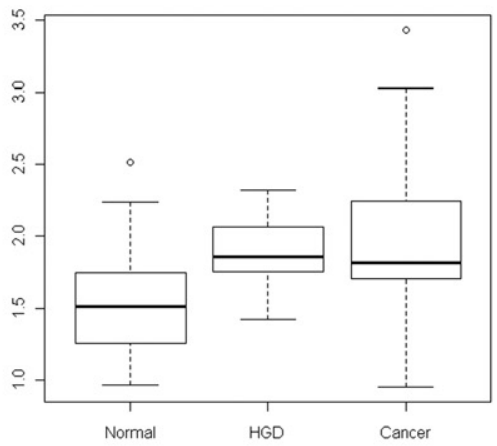

lactate

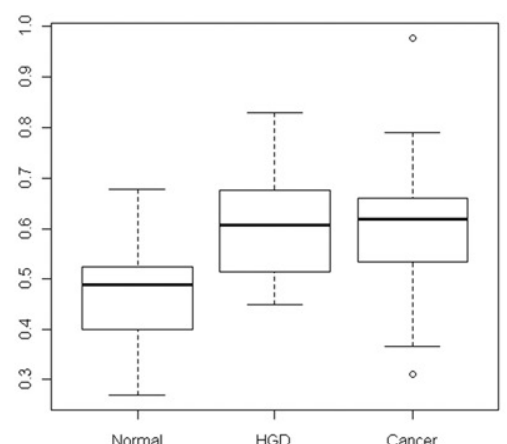

$\beta$-hydroxybutyrate

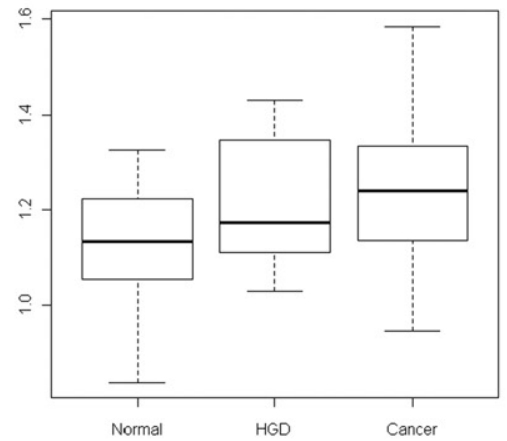

lysine

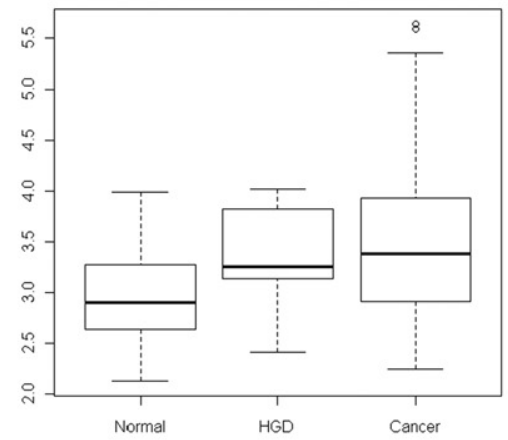

$\alpha$-glucose

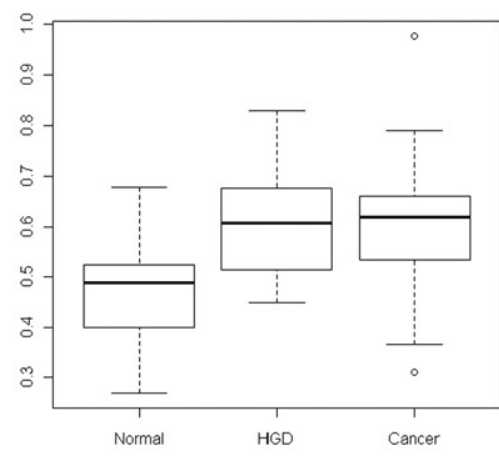

citrate

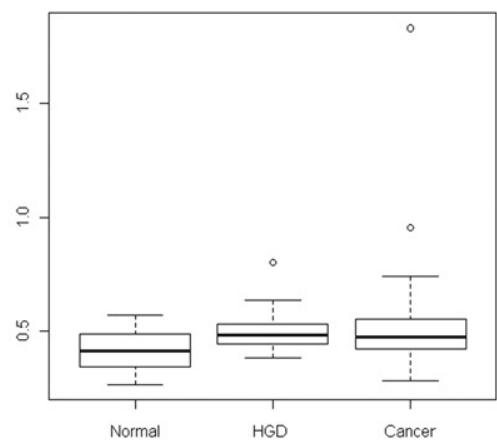

creatinine

FIGURE E2. Box-and-whisker plots of the candidate markers. The y-axis represents the relative concentration level of each metabolite normalized by the internal standard TSP. 
TABLE E1. Summary of spectral regions influencing separation of control samples from cancer samples in the training group.

\begin{tabular}{lcccc}
\hline Chemical shift $(\boldsymbol{\delta})^{*}$ & Multiplicity* & Assignment & $\boldsymbol{P}$ value & Benjamini-Hochberg $\dagger$ correction \\
\hline 1.20 & $\mathrm{~d}$ & $\beta$-hydroxybutyrate & $4.99 \mathrm{E}-03$ & $7.90 \mathrm{E}-03$ \\
1.69 & $\mathrm{~m}$ & Lysine & $6.59 \mathrm{E}-04$ & $1.57 \mathrm{E}-03$ \\
1.91 & $\mathrm{~m}$ & Lysine & $4.61 \mathrm{E}-04$ & $1.25 \mathrm{E}-03$ \\
2.09 & $\mathrm{~m}$ & Glutamine & $2.22 \mathrm{E}-02$ & $3.02 \mathrm{E}-02$ \\
2.31 & $\mathrm{~m}$ & $\beta$-Hydroxybutyrate & $2.25 \mathrm{E}-05$ & $1.42 \mathrm{E}-04$ \\
2.35 & $\mathrm{~m}$ & $\beta$-Hydroxybutyrate & $2.99 \mathrm{E}-03$ & $5.16 \mathrm{E}-03$ \\
2.39 & $\mathrm{~m}$ & $\beta$-Hydroxybutyrate & $2.40 \mathrm{E}-06$ & $2.28 \mathrm{E}-05$ \\
2.53 & $\mathrm{~d}$ & Citrate & $8.70 \mathrm{E}-05$ & $3.31 \mathrm{E}-04$ \\
2.63 & $\mathrm{~m}$ & Unknown & $1.56 \mathrm{E}-06$ & $2.97 \mathrm{E}-05$ \\
2.69 & $\mathrm{~d}$ & Citrate & $6.82 \mathrm{E}-04$ & $1.44 \mathrm{E}-03$ \\
3.00 & $\mathrm{~L}$ & Lysine & $9.63 \mathrm{E}-04$ \\
4.05 & Creatinine & $1.50 \mathrm{E}-04$ & $2.19 \mathrm{E}-02$ \\
4.11 & Lactate & $1.40 \mathrm{E}-03$ & $2.66 \mathrm{E}-03$ \\
5.22 & $\mathrm{~d}$ & $\alpha$-Glucose & $3.15 \mathrm{E}-05$ & $1.49 \mathrm{E}-04$ \\
\hline
\end{tabular}

*The chemical shift and multiplicity are NMR-dependent quantities that indicate the spectral peak position and number of peaks, respectively, that allow the spectroscopist to identify the chemical compound ( $s$, singlet; $d$, doublet; $t$, triplet; $q$, quartet; $m$, complex multiplet). $\dagger$ The Benjamini-Hochberg correction is used to reduce the false discovery rate by adjusting the $P$ value to take into account the use of multiple variable comparisons.

TABLE E2. Student $t$ test results for comparison of various groups

\begin{tabular}{lccccc}
\hline \multicolumn{1}{c}{ Metabolite } & N vs BE & N vs HGD & N vs C & BE vs HGD & BE vs C \\
\hline Glutamine & $1.38 \mathrm{E}-01$ & $3.42 \mathrm{E}-01$ & $3.86 \mathrm{E}-04$ & $6.54 \mathrm{E}-02$ & $2.07 \mathrm{E}-02$ \\
$\beta$-Hydroxybutyrate & $6.62 \mathrm{E}-01$ & $2.39 \mathrm{E}-02$ & $3.34 \mathrm{E}-12$ & $1.54 \mathrm{E}-01$ & $3.08 \mathrm{E}-02$ \\
Citrate & $8.95 \mathrm{E}-01$ & $4.86 \mathrm{E}-03$ & $1.36 \mathrm{E}-07$ & $1.95 \mathrm{E}-01$ & $2.00 \mathrm{E}-01$ \\
Unknown & $4.39 \mathrm{E}-01$ & $1.10 \mathrm{E}-02$ & $4.67 \mathrm{E}-10$ & $5.10 \mathrm{E}-02$ & $1.42 \mathrm{E}-02$ \\
Lysine & $2.36 \mathrm{E}-01$ & $6.77 \mathrm{E}-02$ & $1.99 \mathrm{E}-05$ & $9.96 \mathrm{E}-01$ & 7.01 \\
Creatinine & $8.59 \mathrm{E}-01$ & $2.10 \mathrm{E}-02$ & $5.46 \mathrm{E}-04$ & $1.05 \mathrm{E}-01$ & $9.99 \mathrm{E}-01$ \\
Lactate & $1.74 \mathrm{E}-01$ & $8.21 \mathrm{E}-04$ & $4.82 \mathrm{E}-07$ & $5.29 \mathrm{E}-01$ & $6.09 \mathrm{E}-02$ \\
$\alpha$-Glucose & $3.80 \mathrm{E}-01$ & $2.95 \mathrm{E}-02$ & $2.01 \mathrm{E}-06$ & $5.83 \mathrm{E}-01$ & $6.66 \mathrm{E}-01$ \\
\hline$B E$
\end{tabular}

$B E$, Barrett's esophagus; $C$, esophageal cancer; $H G D$, high-grade dysplasia; $N$, normal sample. 
TABLE E3. $P$ value results for varying genders and ages of the cancer patients

\begin{tabular}{lcc}
\hline \multicolumn{1}{c}{ Metabolite } & M vs F & $\begin{array}{c}\text { Above-average age vs } \\
\text { below-average age* }\end{array}$ \\
\hline Glutamine & 0.494 & 0.888 \\
$\beta$-Hydroxybutyrate & 0.559 & 0.329 \\
Citrate & 0.063 & 0.083 \\
Unknown & 0.395 & 0.935 \\
Lysine & 0.327 & 0.974 \\
Creatinine & 0.054 & 0.118 \\
Lactate & 0.925 & 0.889 \\
$\alpha$-Glucose & 0.855 & 0.344 \\
\hline , male patient; $F$, female patient. *Average age of all cancer patients $=65.6 \mathrm{yrs}$
\end{tabular}

TABLE E4. Absolute concentration comparison of glutamine, creatinine, lactate, and glucose

\begin{tabular}{|c|c|c|c|c|c|}
\hline \multirow[b]{2}{*}{ Metabolite } & \multicolumn{2}{|c|}{ Normal samples } & \multicolumn{2}{|c|}{ EAC samples } & \multirow{2}{*}{$\begin{array}{c}\text { Normal range } \\
(\mathrm{mM})^{*}\end{array}$} \\
\hline & $\begin{array}{l}\text { mean } \\
(\mathrm{mM})\end{array}$ & $\begin{array}{c}\text { SD } \\
(\mathbf{m M})\end{array}$ & $\begin{array}{l}\text { mean } \\
(\mathrm{mM})\end{array}$ & $\begin{array}{c}\text { SD } \\
(\mathbf{m M}) \\
\end{array}$ & \\
\hline Glutamine & 0.469 & 0.054 & 0.515 & 0.068 & $0.502-0.670$ \\
\hline Creatinine & 0.078 & 0.015 & 0.096 & 0.036 & $0.050-0.093$ \\
\hline Lactate & 2.86 & 0.64 & 3.66 & 0.79 & $1.42-4.53$ \\
\hline Glucose & 5.49 & 0.76 & 6.61 & 1.48 & $4.90-5.70$ \\
\hline
\end{tabular}

$S D$, standard deviation. *http://www.hmdb.ca/ 